\title{
Frequency and antibiogram pattern of gram positive cocci in catheter related blood stream infections (CRBSI) in a tertiary care hospital, Tamilnadu
}

\author{
Rajesh J.G ${ }^{1}$, Senthamarai S. $^{2}$, Sivasankari S. $^{3}$, Anitha C.. ${ }^{4}$, Somasunder V. ${ }^{5}$, Kumudavathi M.S. ${ }^{6}$, Akila K ${ }^{7}$, \\ ${ }^{1}$ Dr. Rajesh J.G, Assistant Professor, ${ }^{2}$ Dr. Senthamarai S., Professor, ${ }^{3}$ Dr.Sivasankari S., Associate Professor, ${ }^{4}$ Dr. Anitha \\ C., Assistant Professor, ${ }^{5}$ Dr. Somasunder V., ${ }^{6}$ Dr. Kumuthavathi, Tutor, ${ }^{7}$ Dr. Akila, Assistant Professor, all authors are \\ affiliated with Meenakshi Medical College \& Research Institute, Enathur, Kanchipuram, Tamilnadu, India.
}

Address for Correspondence: Dr. Senthamarai S., Professor, Meenakshi Medical College \& Research Institute, Enathur, Kanchipuram, Tamilnadu, India. Email id: thamaraimicro@gmail.com

\begin{abstract}
Introduction: Blood stream infection associated with catheter is life threatening. Various bacterial agents are responsible for this and Coagulase Negative Staphylococci (CoNS) is now becoming one of the major agent in Catheter Related Blood Stream infection (CRBSI). Objectives: To know the bacterial isolates and the frequency of the CoNS \& its antibiotic resistance patterns of in Catheter Related Blood Stream infection (CRBSI). Methods: Catheter tips (5 cm tip) were collected and $5 \mathrm{ml}$ of peripheral blood sample was collected with proper aseptic precautions. Catheter tips were processed using Maki DG et al procedure. Blood was inoculated into the BACTEC blood culture bottle (BacT/ALERT 3D automated blood culture system -BioMerieux). All the gram positive bacteria were further processed for standard biochemical tests. All these isolates were subjected to antibiotic susceptibility testing (Hi-Media discs) by Kirby Bauer disc diffusion test according to CLSI guidelines. Results: Among the total 158 isolates, 55 (34.8\%) were gram positive cocci. Coagulase Negative Staphylococci (CoNS), were the predominant isolate in this study. Highest resistance was observed with ampicillin, penicillin, amoxyclav, erythromycin and tetracycline. All the gram positive isolates were $100 \%$ sensitive to Vancomycin and Linezolid. Conclusion: Among the total 158 isolates, 55 (34.8\%) were gram positive cocci. Coagulase negative staphylococci (CONS) which account $18.98 \%$, followed by $8.86 \%$, Staphylococcus aureus, $5.06 \%$ Enterococci and $1.89 \%$ were Micrococci. All our isolates were sensitive to Vancomycin and linezolid.
\end{abstract}

Key words: Coagulase Negative Staphylococci (CoNS), Catheter Related Blood Stream Infections (CRBSI), Blood culture.

\section{Introduction}

CRBSI is the presence of bacteria in blood originating from an intravenous catheter which is a life threatening complication of central venous catheterisation [1].

Central venous catheter plays an important role in the management of critically ill patients and pose a risk of device related infection than other devices and cause significant morbidity and mortality [2]. The mortality rate varies between $30-70 \%$ in BSI in hospitalised patients [3]. Gram positive organisms plays a major role in CRBSI than gram negative organism [4]. But both

Manuscript received: $6^{\text {th }}$ January 2017

Reviewed: $16^{\text {th }}$ January 2017

Author Corrected: $24^{\text {th }}$ January 2017

Accepted for Publication: $1^{\text {st }}$ February 2017 gram positive, gram negative bacteria have been isolated from CRBSI and predominance of one type over others varies from place to place and even in the same place over time [5]. Isolation of the pathogen and determination of antimicrobial susceptibility pattern of the isolates remain the anchor of definitive diagnosis and management of BSI [6].

The emergence of antibiotic resistance in all kinds of bacteria is a serious public health issue worldwide, which could be due to increased inappropriate antibiotic use or misuse. The reports of wider variation in the resistance pattern in one area in one period of time are not necessarily true for other area or period [7]. 
Hence, this study was conducted to investigate the frequency of bacterial pathogens causing catheter associated BSIs in our hospital and to know about antimicrobial resistance in order to help clinicians to choose the most suitable therapy.

\section{Materials and Method [8, 9, 10]}

Study design: Descriptive study

Setting: This study was conducted in microbiology department, Meenakshi Medical College \& Research Institute, Kanchipuram. The study period was from March 2014 to August 2015. Ethical clearance was obtained from institution ethical committee.

Inclusion Criteria: Patients with signs and symptoms of septicaemia after 48 hours of venous catheterisation were included in this study. Brief personnel and epidemiological data were collected from the patients after getting the informed consent.

Exclusion criteria: Patients who already had signs and symptoms of septicaemia at the time of admission and less than 48 hours of venous catheterisation were excluded in this study.

Study size \& Participants: A total of 812 catheter tips (5 cm tip) were collected and $5 \mathrm{ml}$ of peripheral blood sample was collected with proper aseptic precautions.
Method: Catheter tips were processed using Maki DG et al procedure. Blood was inoculated into the BACTEC blood culture bottle (BacT/ALERT 3D automated blood culture system - BioMerieux). From the positive BacT bottles, it was sub cultured on blood agar, nutrient agar, chocolate agar, \& MacConkey agar and further incubated at $37^{\circ} \mathrm{c}$ overnight and the colony morphology was examined and subjected for gram staining.

All the gram positive bacteria were further processed for standard biochemical tests. Among 812 catheter tips, $158(19.75 \%)$ were found to be culture positive. In this 158 positive isolates, 55 were gram positive cocci $(34.8 \%)$ All these gram positive isolates were subjected to antibiotic susceptibility testing (Hi-Media discs) by Kirby Bauer disc diffusion test according to CLSI guidelines.

Variables: Of 55 gram positive cocci, 34 were male and 21 were female.

Data source: Intensive care unit (ICU) patients after 48 hours with signs \& symptoms of septicaemia.

Bias: we eliminated the bias by applying simple random sampling method for selecting the subject from ICU.

Statistical methods: Chi-square test $\left(\chi^{2}\right)$ was applied to find if any equal distribution in the type of CRBSI.

\section{Results}

Out of the total 812 blood samples, $158(19.75 \%)$ isolates were found to be positive with CRBSI. Among the total 158 isolates, $55(34.8 \%)$ were gram positive cocci. Among 158 isolates, 27 isolates grown either in blood culture or in catheter tip which were excluded.

Table-1- Distribution of Gram Positive Cocci (n=55)

\begin{tabular}{|c|c|c|}
\hline Gram positive organism & No of isolates & \% CRBSI \\
\hline Coagulase negative staphylococci (CONS) & 30 & $54.5 \%$ \\
\hline Staphylococcus aureus & 14 & $25.45 \%$ \\
\hline Enterococci & 8 & $14.54 \%$ \\
\hline Micrococci & 3 & $5.45 \%$ \\
\hline Total & $\mathbf{5 5}$ & $\mathbf{1 0 0} \%$ \\
\hline
\end{tabular}

Among the gram positive cocci, the predominant organism of isolation were Coagulase negative staphylococci (CONS) followed by Staphylococcus aureus.

The table value is 7.82 for $3 \mathrm{df}$ and at $\mathrm{P}=0.05 . \chi^{2}$ Calculated $=13.01>7.82$, So, there is significant difference between observed frequencies and expected frequencies. Hence we reject null hypothesis. Therefore we infer that the distribution of cases to infections vary from one another. 
Research Article

Table-2: Sex wise distribution of Gram Positive Cocci among CRBSI $(\mathbf{n}=55)$

\begin{tabular}{|c|c|c|}
\hline $\mathbf{n = 5 5}$ & Number & Percentage (\%) \\
\hline Male & 34 & 61.8 \\
\hline Female & 21 & 38.2 \\
\hline
\end{tabular}

Among the sex distribution, $61.8 \%$ were male and $38.2 \%$ were female.

Table-3: Antibiotic Resistance pattern of Gram Positive Cocci among CRBSI (n=55).

\begin{tabular}{|c|c|c|}
\hline Antibiotics (mcg) & Number (n=55) & Percentage (\%) \\
\hline Ampicillin (30) & 45 & 78 \\
\hline Penicillin & 43 & 72 \\
\hline Amoxyclav (30) & 41 & 69 \\
\hline Gentamycin & 38 & 69 \\
\hline Erythromycin (15) & 38 & 49 \\
\hline Tetracycline (30) & 27 & 27 \\
\hline Cotrimoxazole (25) & 18 & 4 \\
\hline Ceftazidime (30) & 15 & 0 \\
\hline Cephalexin (30) & 2 & 0 \\
\hline Oxacillin & 0 & 33 \\
\hline Linezolid (30) & 0 & 75 \\
\hline Vancomycin (30) & 27 & \\
\hline
\end{tabular}

Among the gram positive isolates, highest resistance $81 \%, 78 \%, 75 \%, 72 \%, 69 \%$ and $69 \%$ were observed with ampicillin, penicillin, amoxyclav, gentamycin, erythromycin, and tetracycline respectively. All our isolates were sensitive to Vancomycin and linezolid. 4\% of gram positive isolates were resistant to oxacillin.

\section{Discussion}

In blood stream infections, bacteriological profile and antimicrobial susceptibility pattern plays an important role in effective management. In our study, $19.75 \%$ showed the positive growth in culture. Many authors have observed the rates of $17 \% \quad 18.6 \%$ \& $21.7 \%$ respectively in their studies $[11,12,2]$. Our study is in line with these studies. Vanitha Rani et al., [7] has reported a lower rate of $8.39 \%$ whereas $44.9 \%$ was the incidence rate in the study of Martins et al [13]. The results have varied with various studies could be due to different factors like socioeconomic, use of ventilators and practice of antibiotic in that locale.

Among the blood culture, $34.8 \%$ were gram positive cocci in our study. It was documented that the rates of gram positive cocci isolation in blood culture were, 37.7 $\%, 41.9 \%$ \& $69.0 \%$ with various studies respectively $[7$, $12,14]$. Our study is slightly lower than these studies. Male were commonly affected $(61.8 \%)$ in our study.
Among the gram positive cocci, Coagulase Negative Staphylococci (CoNS), were the predominant isolate in this study. This is followed by Staphylococcus aureus (8.86\%), Enterococci (5.06\%) and Micrococci (1.89\%).

Though CoNS have been considered as non-pathogenic, due to the both increased use of intravascular device and immune compromised population, it is considered as major nosocomial pathogens. CONS were the major isolates with various studies $[15,16]$. Staphylococcus aureus was the predominant organism in various studies $[2,12,7]$.

All the gram positive cocci were subjected for antimicrobial susceptibility test. In our study, highest resistance was observed with ampicillin, penicillin, amoxyclav, erythromycin and tetracycline. Similar high resistance of ampicillin was observed by many authors $[12,7,17,18]$. 
Research Article

Highest resistance for Erythromycin was reported by Ashwini et al, whereas it was highly sensitive agent in the study of Meenakshi et al., [19]. 95\% \& 90\% of penicillin resistance were noted in some studies [20,21]. The variation in resistance pattern with different studies are due to the environment where the test is done and use of newer technology and the pattern of antibiotic practice in that locale. Regular monitoring of pathogen and update of antibiotic resistant pattern is very important in prevention and management of infection.

In our study the methicillin resistance rate was very less (4\%). But high methicillin resistance rate of $87.5 \%$, was noted by Kaur et al. $26.7 \%$ of methicillin resistance was observed by Parameswaran et al [2, 22]. Methicillin resistant gram positive isolates are increasingly reported in various studies and it is a dominant and significant threat to human.

All the gram positive isolates were $100 \%$ sensitive to Vancomycin and Linezolid. This in line with many studies. [17, 23]. Glycopeptides have been considered to be the reserve drug for these organism. In our study, cephalosporin group of drugs are promising.

\section{Conclusion}

The present study provided the prevalence of gram positive cocci in causing blood stream infections and their antibiotic sensitivity pattern in our locale. In each area, the determination of organism responsible for BSI and the antibiotic resistant pattern in periodic interval is necessary for the clinicians to be aware of the pathogens and to provide safe and effective therapy. So that we can develop rational prescribing practices and formulate antibiotic policy.

Acknowledgement- We acknowledge Mr. Balasubramanium, Statistian, Department of Social and Preventive Medicine, our college, for the statistical analysis.

Funding: Nil, Conflict of interest: None initiated, Permission from IRB: Yes

\section{References}

1. Mermel LA. Prevention of intravascular catheterrelated infections. Ann Intern Med. 2000 Mar 7;132 (5): 391-402.

2. Kaur M, Gupta V, Gombar S, Chander J, Sahoo T. Incidence, risk factors, microbiology of venous catheter associated bloodstream infections--a prospective study from a tertiary care hospital. Indian J Med Microbiol. 2015 Apr-Jun;33(2):248-54. doi: 10.4103/0255-0857. 153572.

3. Vincent JL, Bihari DJ, Suter PM, Bruining HA, White J, Nicolas-Chanoin MH, Wolff M, Spencer RC, Hemmer M. The prevalence of nosocomial infection in intensive care units in Europe. Results of the European Prevalence of Infection in Intensive Care (EPIC) Study. EPIC International Advisory Committee. JAMA. 1995 Aug 23-30;274(8):639-44.

4. Eggimann P, Pittet D. Overview of catheter related blood stream infections with special emphasis on prevention based on educational programs. Clin microbial infect 2002; 8:295-309.

5. Akingbade OA, Ojo DA, Okerentuba PO, Adejuwon AO, Okonko I O. Antibiotic resistant profile of bacteria isolated from septicaemia cases in a tertiary health care in Abeokuta, Nigeria. Nature and science 2013:11(2)

6. Qureshi M, Aziz F. Prevalence of microbial isolates in blood culture and their antimicrobial susceptibility profile. Biomedica 2011; 27: 136-39.

7. Vanitha Rani N, Kannan Gopal, Venkata Narendra M, Viswakanth D, V R D Nagesh, YohithaM, Venkatasunil M, ThennarasuPalani. A retrospective study on blood stream infections and antibiotic susceptibility patterns in a tertiary care teaching hospital. International journal of pharmacy and pharmaceutical sciences. Vol 4, 1:2012.

8. Bailey \& Scottt's Diagnostic microbiology.13 ${ }^{\text {th }}$ edition, Elsevier publications.

9. Maki DG, Weise CE, Sarafin HW. A semiquantitative culture method for identifying intravenous-catheter-related infection. N Engl J Med. 1977 Jun 9; 296(23):1305-9.

10. Clinical and Laboratory Standards Institute. Performance standards for antimicrobial susceptibility testing; CLSI, 2014.

11.S.B. Mishra, R. Misra, A. Azim, A.K. Baronia, K.N. Prasad, T.N. Dhole, M. Gurjar, R.K. Singh, B. Poddar. Incidence, risk factors and associated mortality of central line-associated bloodstream infections at an 


\section{Research Article}

intensive care unit in northern India. Int $\mathbf{J}$ Qual Health Care. 2016:29(1). Doi:10.1093/intqhe/mzw144.

12. Ashwini Saminder Waghmare, M.HimaBindu, C. Mallikarjuna Reddy. Bacterial isolates and antibiotic susceptibility pattern in blood stream infections suspected patients attending a teaching hospital in Telungana, India. Int.J.Curr.Microbiol.App.Sci (2015) 4 (7): 741-748.

13. Martins M.M, Chukwuemeka E.A, Anne E.A, Joseph U.O, Simon.E A. Bacterial isolates from blood cultures of children with suspected septicaemia in calbar Nigeria. BMC Infectious Diseases: 2005: 5:110.

14. Mulat Dagnew, GizachewYismaw ,Mucheye Gizachew, Alemayehu Gadisa, Tigist Abebe, Tinebeb Tadesse, Agersew Alemu and Biniam Mathewos. Bacterial profile and antimicrobial susceptibility pattern in septicemia suspected patients attending Gondar University Hospital, Northwest Ethiopia, January 2013:6 (283):1-7

15. Richard S. Johannes, Marlborough, Massachusetts. Epidemiology of early-onset bloodstream infection and implications for treatment. American journal of infection control December 2008:Volume 36, Issue 10, Pages S171- e13- e17.doi:http://dx.doi. org/10.1016/j. ajic.2008.10.003.

16. K Chopdekar, C Chande, S Chavan, $\mathrm{P}$ Veer, V Wabale, K Vishwakarma, A Joshi. Central venous catheter-related blood stream infection rate in critical care units in a tertiary care, teaching hospital in Mumbai. Year : 2011|Volume : 29 | Issue : 2 |Page : 169-171.
17. Surekha.Y.Asangi, Mariraj. J, Sathyanarayan.M.S, Naghabhushan, Rashmi. Speciation of clinically significant Coagulase Negative Staphylococci and their antibiotic resistant patterns in a tertiary care hospital. Int J Biol Med Res: 2011:2(3):735-739.

18. Shubhra Singh, Gopa Banerjee et al. Prevalence of MecA gene positive Coagulase Negative Staphylococci in NICU of tertiary care hospital. Biomedical Research. 2009:20(2); 94-98.

19. Meenakshi Kante, Muni Lakshmi P, Sreenivasulu Reddy P. Bacterial profile of blood stream infections and their antibiograms. Int J Res Med Sci. 2015; 3(3): 698-704

20. I. Roy, A. Jain, M. Kumar, and S. K. Agarwal. Bacteriology of neonatal septicemia in a tertiary care hospital of Northern India. Indian Journal of Medical Microbiology, vol. 20, pp. 156-159, 2002.

21. Jyoti Pal, Dakshina Bisht. Characterization and Risk Factors of Candida Species in Nosocomial Blood Stream Infections. Santosh University Journal of Health Sciences 2015; 1(2):57-59.

22. Parameswaran R, Sherchan JB, Varma D M, Mukhopadhyay C, Vidyasagar S. Intravascular catheterrelated infections in an Indian tertiary care hospital. J Infect Dev Ctries. 2011 Jul 4;5(6):452-8.

23. Sharma PP, Halder D, Dutta AK, Dutta R, Bhatnagar S, Bali A, Kumari S. Bacteriological profile of neonatal septicemia. Indian Pediatr. 1987 Nov;24 (11):1011-7.

\section{How to cite this article?}

Rajesh J.G, Senthamarai S, Sivasankari S, Anitha C, Somasunder V, Kumudavathi M.S, Akila K, Frequency and antibiogram pattern of gram positive cocci in catheter related blood stream infections (CRBSI) in a tertiary care hospital, Tamilnadu. Trop J Path Micro 2017;3(2):102-106.doi: 10.17511/jopm.2017.i2.04. 